\title{
L'écologie humaine de Pierre Dansereau et la métaphore du paysage intérieur
}

\author{
René Audet \\ Sociologue, Chaire de responsabilité sociale et de développement durable, Université du Québec à Montréal, Montréal, Québec,
} Canada H3C 3P8

\begin{abstract}
Dans la démarche scientifique de l'écologue Pierre Dansereau, décédé en septembre 2011 à l'âge de 99 ans, l'homme n'a jamais été très éloigné de la nature, comme il l'expliquait dans un entretien accordé à NSS $(12,1$ [2004]) à propos de son traité de biogéographie. Et c'est certainement le positionnement éthique et épistémologique de ce précurseur des approches interdisciplinaires qui l'a conduit notamment à s'intéresser au champ de l'écologie humaine.

Cette question de l'écologie humaine permet en effet de s'interroger sur le lien possible entre les modèles de l'écologie et le fonctionnement des sociétés humaines. À défaut d'apporter une réponse à ce questionnement, le texte de René Audet nous montre en quoi, dans l'itinéraire scientifique de P. Dansereau, on retrouve l'invention, comme le disait si bien Michel de Certeau à propos du quotidien, de mille manières de braconner.
\end{abstract}

La Rédaction

\section{Mots-clés :}

Pierre Dansereau ; écologie humaine ; métaphore ; naturalisme ; représentations sociales

\begin{abstract}
Résumé - La renommée de Pierre Dansereau n'est plus à faire dans le monde des sciences de l'environnement, de l'écologie et de l'écologie humaine. Sa disparition récente, à quelques jours de son centenaire, à soulevé une vague de reconnaissance pour sa longue carrière et son grand engagement humaniste. $C^{\prime}$ est dans ce contexte qu'est ancré le propos du présent article. Nous entreprenons ici d'examiner la pertinence scientifique de l'écologie humaine de Dansereau à la lumière de deux défis épistémologiques rencontrés par toute science s'intéressant aux rapports des humains à leur environnement : l'usage des modèles explicatifs «naturalistes » et l'utilisation de métaphores. Les métaphores naturalistes, affirmons-nous, donnent une image trop simpliste de la dimension symbolique du rapport à la nature. Après avoir examiné les deux principales métaphores naturalistes qui ont marqué l'écologie, nous montrons que l'écologie humaine de Dansereau, si elle est bien ancrée dans l'une d'elles, ne fait pas l'erreur d'ignorer l'importance des représentations symboliques de l'environnement, qu'il introduit par la belle métaphore du paysage intérieur.
\end{abstract}

\section{Keywords:}

Pierre Dansereau; human ecology; metaphors; naturalism; social representations

\begin{abstract}
Pierre Dansereau's human ecology and the inscape metaphor. Pierre Dansereau's reputation is well established in the world of environmental sciences, and more specifically in ecology and human ecology. The man's recent passing, a few days before his centennial, has brought up a wave of recognition for his long career and great humanist engagement. In this paper, we explore the scientific side of his work in human ecology. Our goal is to measure its actual scientific relevance in the light of two related epistemological challenges faced by all sciences concerned with the nature-culture dualism, that is the use of naturalistic explanations and that of metaphors. Naturalist metaphors, we argue, are over simplistic regarding the complexity of the symbolic dimension of the human-nature relationship. After an evaluation of the pitfalls of the principal metaphors in the sciences of ecology and human ecology, we show that while Dansereau's human ecology is anchored into one of them, it avoids the fallacy that consists of ignoring the importance of symbolic representations in our relation to the environment. P. Dansereau does so by introducing the beautiful metaphor of the inscape.
\end{abstract}




\section{Introduction}

$\mathrm{Au}$ Québec, Pierre Dansereau est surtout connu comme pionnier de l'écologie et du travail interdisciplinaire en environnement. En prenant connaissance de sa contribution à la discipline de l'écologie humaine, on découvre également le grand engagement humaniste de " l'écologiste aux pieds nus ${ }^{1}$ ». À l'heure du centenaire de cet homme et, simultanément, de sa disparition (le 29 septembre 2011), alors que se multiplient les hommages à sa personnalité et à son apport à l'avancement de l'écologie au Québec et dans le monde (Dupont, 2010 ; UQAM, 2010 ; Dansereau et al., 2004 ; Vaillancourt, 1999), nous entendons ici revenir plus en profondeur sur la pertinence scientifique actuelle de l'œuvre de Dansereau, et plus particulièrement de son écologie humaine ${ }^{2}$.

Il suffit de jeter un coup d'œil sur le parcours professionnel de Dansereau pour comprendre l'envergure de l'homme. Fondateur du service de biogéographie de l'Université de Montréal dans les années quarante, il devient en 1950 professeur d'écologie à l'Université Ann Arbor (Michigan), où il rédigera son principal ouvrage d'écologie scientifique (Biogeography: An Ecological Perspective [Dansereau, 1957]), puis revient à l'Université de Montréal en 1955 comme doyen de la faculté des arts. C'est dans les années soixante, alors qu'il s'installe à New York pour enseigner à l'Université Colombia et codiriger le jardin botanique de New York, que Dansereau commencera véritablement à élaborer son écologie humaine. Il reviendra à l'Université de Montréal en 1968, puis passera à l'Université du Québec à Montréal en 1971, où il sera nommé professeur émérite en 1989. Bien qu'un chapitre de Biogeography traitât déjà en 1957 de l'impact de l'être humain sur les écosystèmes, c'est en 1973 que fut publié, en français, son principal ouvrage d'écologie humaine: La Terre des hommes et le paysage intérieur (Dansereau, 1973). Mi-savante, mi-vulgarisée, l'écologie humaine présentée dans ce livre est reprise et précisée dans une série d'articles scientifiques qui, des années 1960 aux années 1990, ont occupé les recherches définitivement interdisciplinaires de Dansereau.

L'écologie humaine de Dansereau est représentative d'une tendance des années 1970 qui amenait des écologues et des sociologues à travailler de pair pour fonder un « nouveau paradigme » interdisciplinaire, axé sur le rapprochement entre les approches plus interprétatives des sciences sociales et les approches plus explicatives de l'écologie (Catton et Dunlap, 1980 ; Dunlap, 2008). L'éco-

\footnotetext{
1 Nous faisons référence à la biographie rédigée par Thérèse Dumesnil (1981).

2 Une version antérieure de cet article a fait l'objet d'une présentation au $18^{\mathrm{e}}$ congrès de l'Association internationale des sociologues de langue française, à Istanbul en juin 2008.
}

logie humaine, à cause de cette position particulière au croisement des sciences sociales et naturelles, se doit de considérer la matrice épistémologique de l'interprétation moderne de la relation entre l'être humain et les écosystèmes que l'on a appelée la «dualité nature-culture » (Latour, 1997). Klaus Eder (1996, p. 7) donne un aperçu du débat porté par cette dualité en affirmant :

"The relationship between nature and society can be conceived of theoretically in two mutually exclusive ways: as a natural constitution of society or as a social construction of nature. These alternatives divide the theoretical discourse on the relationship between nature and society into two camps: a naturalist and a culturalist camp. »

Le défi, d'ailleurs toujours très actuel, de trouver un langage commun aux sciences de la nature et aux sciences sociales ne va donc pas sans difficulté, et toutes les tentatives visant à accomplir cette mythique "synthèse des disciplines » se sont frottées aux problématiques épistémologiques et méthodologiques émanant de cette dualité. Il convient donc, en guise d'explication préliminaire, d'identifier ces problématiques qui baliseront notre lecture de l'écologie humaine de Dansereau dans la suite de cet article. Nous en analyserons deux.

La première, de nature plus épistémologique, porte sur les écueils des explications trop naturalistes de la vie sociale. L'écologie humaine a traditionnellement tiré des disciplines de l'écologie et de la biologie sa tendance à expliquer les faits sociaux dans un cadre naturaliste (Lentin, 1977), ce qui implique nécessairement un certain degré de réductionnisme à l'égard de ce que l'on pourrait appeler la «dimension symbolique de la vie sociale» - c'est-à-dire l'aspect interprétatif, les faits idéologiques et culturels, etc. Nous chercherons donc à évaluer si Dansereau, malgré les fondements naturalistes de sa discipline, a mieux réussi que d'autres à intégrer la dimension symbolique de la vie sociale à son écologie humaine.

La deuxième problématique est de nature plus méthodologique et elle est en quelque sorte liée à la première : il s'agit des simplifications à outrance entraînées par l'utilisation de métaphores tirées de la biologie ou des sciences physiques dans la description et l'explication de l'objet de l'écologie humaine, à savoir les interactions entre les dynamiques sociales et les écosystèmes. Dans son ouvrage sur l'histoire de l'écologie, Jean-Marc Drouin (1993, pp. 129-130) explique :

« N'ayant jamais en droit valeur démonstrative, les analogies et les métaphores se retrouvent ou en amont ou en aval del'administration de la preuve : en amont, dans l'invention de l'hypothèse ; en aval, dans la communication didactique ou la réflexion philosophique. Les repérer permet d'accéder à la dimension imaginaire de l'activité scientifique [...]. Des préférences affectives, esthétiques, mais aussi politiques semblent souvent influencer l'usage ou le rejet d'une analogie. Ceci se vérifie en écologie comme ailleurs. » 
L'utilisation de métaphores, si elle peut avoir une valeur heuristique, mène souvent à des simplifications de la réalité - des " réductionnismes ». Dans le cas des métaphores de l'écologie, c'est la réalité sociale, avec toute sa dimension symbolique, qui risque le plus souvent d'être amputée d'une partie importante de sa complexité par l'usage de métaphores naturalistes. Les deux premières parties de cet article s'attardent d'ailleurs à présenter les deux principales métaphores élaborées par l'écologie et l'écologie humaine au $X X^{\mathrm{e}}$ siècle : la métaphore organiciste et la métaphore systémique. L'écologie humaine de Dansereau comporte-t-elle ce type de métaphore, qui tendrait à réduire sa capacité d'analyse fine des faits sociaux? Nous verrons dans les troisième et quatrième parties de ce texte que, si cette écologie humaine ne réussit pas à s'affranchir d'une approche naturaliste et si ellen'est pas non plus exempte de raisonnement métaphorique, elle montre tout de même une certaine conscience des problèmes que cela entraîne et tente $d^{\prime} y$ remédier en renvoyant à l'horizon éthique du « paysage intérieur».

\section{L'écologie humaine organiciste du groupe de Chicago}

Au début du XXe siècle, l'écologie humaine naît ellemême d'une métaphore empruntée à la jeune science de l'écologie. Il est d'ailleurs intéressant de noter que, contrairement à la deuxième écologie humaine, qui fut le produit d'écologues (comme Dansereau) s'intéressant à l'organisation humaine, cette première écologie humaine se développa à partir des sciences sociales. Avant le triomphe de l'approche interactionniste symbolique que l'on connaît en sociologie sous l'étiquette de «l'école de Chicago ", des chercheurs de l'Université de Chicago avaient déjà, avec l'objectif d'étudier le développement urbain effréné du début du siècle, élaboré une écologie humaine inspirée de l'état des connaissances en écologie. Ce groupe de sociologues et de géographes, que l'on peut appeler le « groupe de Chicago » pour le distinguer de l'école sociologique qui lui a succédé (Acot, 1994), définissait l'écologie humaine comme « l'étude des relations spatiales et temporelles des êtres humains en tant qu'affectées par des facteurs de sélection, de distribution et d'adaptation liés à l'environnement » (McKensie, 1979, p. 150). À lire les principaux textes du groupe de Chicago, on constate facilement l'emprunt d'une terminologie propre aux sciences naturelles de l'époque - emprunt qui donne d'ailleurs lieu aux glissements et distorsions traduisant une connaissance rudimentaire de ces sciences. Ainsi, non seulement les « relations spatiales et temporelles » que ces chercheurs voulaient étudier étaient-elles affectées par des " facteurs de sélection et d'adaptation ", mais elles impliquaient aussi toute une série de considé- rations sur la « nature humaine » et son statut $\mathrm{d}^{\prime}$ « animal grégaire », sur les « cycles » du développement urbain et leurs «facteurs perturbants », et plus généralement sur "l'organisation écologique de la communauté humaine » et l'atteinte d'un éventuel "climax» (Grafmeyer et Joseph, 1979). Plus qu'un emprunt de terminologie, l'écologie humaine du groupe de Chicago constituait une tentative de transposition de la métaphore de l'écologie organiciste à la société.

L'écologie organiciste fut principalement élaborée, au tournant $\mathrm{du} X \mathrm{X}^{\mathrm{e}}$ siècle, dans les travaux de Frederic E. Clements, un écologue qui observait les transformations et les successions des " communautés biotiques" dans les prairies de l'Ouest américain, alors en cours de mise en culture massive pour la production de céréales. Clements assimilait son unité d'analyse, la communauté biotique, à un organisme complexe doté d'une relative autonomie, possédant fonctions et structures et évoluant dans le temps à travers divers stades de développement, del'enfance à la maturité ${ }^{3}$. La métaphore organiciste avait donc ici une valeur à la fois heuristique et analytique, puisqu'elle était posée comme un véritable cadre théorique pour l'écologie (Deléage, 1991). Selon ce modèle, le développement des formations végétales s'effectuait dans un ordre déterminé qui, malgréles effets des facteurs physiques et environnementaux externes à la communauté végétale, comme les phénomènes d'invasion, allait nécessairement évoluer vers «le niveau le plus élevé possible sous les conditions climatiques présentes » (Clements, 1928, p. 4) ${ }^{4}$. L'atteinte éventuelle du climax, idée puisée dans la jeune tradition de l'écologie américaine de l'époque et qui renvoyait à l'état d'équilibre et de reproduction d'une formation végétale résultant de l'achèvement des phases de succession et de l'influence des conditions géoclimatiques externes, venait coiffer le cycle du développement de ces formations.

C'est donc cette conception de la succession des espèces qui allait inspirer l'écologie humaine du groupe de Chicago. Le résultat de la transposition de la métaphore organiciste à la société se manifesta tant dans le langage emprunté à l'écologie que dans les interprétations naturalistes d'une urbanisation désordonnée et ségrégative. Les phénomènes de l'exode rural et de l'intensification démographique des villes industrielles du début du siècle engendraient de nouveaux problèmes sociaux urbains que le groupe de Chicago tentait de comprendre d'un point de vue macrosociologique, fonctionnaliste et déterministe. Dans leurs travaux, ces savants ont ainsi classé les communautés humaines selon

\footnotetext{
3 Clements (1928,p. 3) affirme : « As an organism the formation arises, grows, matures, and dies. Its response to the habitat is shown in processes or functions and in structures which are the record as well as the result of these functions. "

4 Notre traduction.
} 
leurs fonctions productives principales et montré en quoi la complémentarité des fonctions des communautés les rendait interdépendantes, comme le sont les organes du corps ou les espèces d'une formation végétale. Dans le grand corps qu'est la société, on pensait que chaque communauté pouvait atteindre un état de stabilité et d'équilibre, son "climax», jusqu'à ce qu'un élément, comme l'arrivée d'un nouveau moyen de transport, l'épuisement ou la découverte de ressources, l'innovation technologique, etc., ne vienne perturber cet ordre. Ainsi, «le développement structuré de la communauté se fait selon une séquence qui n'est pas sans rappeler les étapes successives du développement de la formation végétale » (McKensie, 1979, p. 161).

En somme, la société américaine en cours de transformation au début du siècle posait de nouveaux défis à l'ordre social et le groupe de Chicago se donnait donc la mission d'identifier les mécanismes et les facteurs de sélection, de distribution et d'adaptation qui la ramèneraient vers l'équilibre d'un nouvel ordre social. À cette approche organiciste de la première écologie humaine, il faut poser deux principales objections. Premièrement, la linéarité mécanique et déterminée que suppose l'idée de climax empêche toute analyse de l'action sociale. Le processus de développement de la ville est considéré comme inéluctable, sans égard aux actions entreprises pour le contrer, en limiter les impacts ou le contourner. Ainsi McKensie (loc. cit., p. 158) affirme-t-il que, dans certaines communautés touchées par l'exode vers la ville, «des réformateurs s'efforcent en vain d'amener les jeunes à rester à la ferme ou dans leur village natal, sans se rendre compte qu'ils vont ainsi à contre-courant des principes généraux de l'ordre écologique». Sans avoir besoin de citer l'une ou l'autre des approches sociologiques développées au cours du dernier siècle, il faut convenir que ce genre de déterminisme absolu est exagérément réducteur.

La deuxième objection concerne les implications morales de la métaphore organiciste. La transposition de « lois » de la nature aux phénomènes sociaux entraîne, en effet, certaines conclusions dangereusement simplistes. Par exemple, lorsqu'il décrit les processus écologiques déterminant la structure interne de la communauté, McKensie n'hésite pas à comparer l'arrivée de nouvelles populations à des « invasions » parasitaires : « on observe communément que les races étrangères et autres envahisseurs indésirables, à quelques exceptions près, élisent domicile près du centre $d^{\prime}$ affaires ou en d'autres points de forte mobilité et de faible résistance » (ibid., p. 163). Les problèmes sociaux qui suivent 1 'industrialisation, comme la ségrégation raciale, la violence et sa répression, l'isolement de certains groupes sociaux ou culturels, apparaissent comme des phénomènes «naturels » ou « écologiques ». Ces problèmes ne sont donc pas considérés immoraux, ce qui fait dire à Acot (1994, p. 90) que « cette métaphore est d'une extrême violence : ou bien la ville digère ses immigrés, ou bien elle les expulse comme des déchets $[\ldots]$ ».

Dansereau investira lui aussi l'objet de recherche qu'est l'écosystème urbain, mais ne succombera pas à la métaphore organiciste. Toutefois, cela ne signifie pas que ses travaux excluent toute métaphore. Dansereau s'inscrira dans un courant qui, appliqué à l'écologie, introduit la métaphore des systèmes dans l'étude des milieux de vie, puis des milieux humains.

\section{L'écologie systémique}

Il est ironique que ce soit Arthur Tansley, l'un des plus critiques dénonciateurs de la métaphore organiciste, qui ait inventé le mot « écosystème », lui-même inspiré d'une autre métaphore, systémiste celle-là. Dans son célèbre article intitulé "The use and abuse of vegetational concepts and terms ", Tansley (1935) rassemblait ses critiques de l'approche organiciste et remettait en cause les concepts de succession et de développement qui donnaient, selon lui, une image trop unidirectionnelle des changements qui s'opèrent dans les écosystèmes ; celui de climax, trop peu subtil pour illustrer les différents facteurs qui peuvent affecter l'équilibre d'une association végétale ; et, surtout, celui " $\mathrm{d}$ 'organisme complexe", dérivé d'une croyance holiste et vitaliste non fondée objectivement. Ainsi Tansley propose-t-il de se débarrasser de la notion d'organisme et de s'intéresser plutôt « à la totalité du système » en incluant non seulement les éléments organiques, mais aussi tous les facteurs physiques dans une unité d'analyse rebaptisée " écosystème », « qui est une catégorie particulière parmi les systèmes physiques qui constituent l'univers » (ibid., p. 306) ${ }^{5}$.

Depuis, lorsque les écologues parlent de système, c'est bien des systèmes de la physique et des lois de la thermodynamique qu'ils s'inspirent. Peu de temps après la publication de l'article fondateur de Tansley, Raymond Lindeman et G. Evelyn Hutchinson ont développé leur approche trophique-dynamique, qui consistait à décrire les fonctions et les interactions des écosystèmes en termes d'échange d'énergie, notamment en soumettant ces écosystèmes aux lois de la conservation et de la transformation de l'énergie (Deléage, 1991). L'approche systémique devient alors un puissant cadre théorique pour l'écologie et, éventuellement, pour l'ensemble des sciences de l'environnement :

« Inspiré des notions de rétroaction, de causalité circulaire et d'information, Hutchinson développe un modèle écologique dans lequel les environnements naturels sont conçus comme des réseaux interdépendants en constante relation les uns avec les autres. C'est à un de ses étudiants de Yale,

Notre traduction. 
[Howard T.] Odum, que l'on doit l'élaboration du concept d'écosystème. Ce dernier participe d'une naturalisation du modèle cybernétique à l'échelle planétaire. La nature tout entière devient ainsi un immense système cybernétique. " (Lafontaine, 2004, pp. 76-77.)

Howard T. Odum et son frère Eugene P. Odum sont connus pour avoir contribué à l'écologie systémique de manière déterminante, en comptant les écosystèmes parmi les unités du domaine du vivant capables d'éviter l'état "d'entropie », c'est-à-dire l'arrêt des échanges d'énergie dans un système après la dissipation progressive de l'énergie (ibid.). H.T. Odum, plus particulièrement, a ajouté aux quatre lois de la thermodynamique le « principe du maximum de puissance » qui stipule que « les écosystèmes, qui ont des aptitudes particulières à l'utilisation de la puissance des flux d'énergie, sont aussi les plus aptes à la survie et à la domination » (Deléage, 1991, p. 137). Ainsi, l'écologie systémique des frères Odum contribua-t-elle à la diffusion du langage de la thermodynamique et de la métaphore systémiste en écologie. Ils nes'arrêtèrent d'ailleurs pas auxécosystèmes " naturels » et soutinrent que les lois de l'écologie systémique devaient s'appliquer tant aux systèmes sociaux qu'aux écosystèmes, où les principes d'utilisation maximale et de conservation d'énergie sont aussi à l'œuvre. Par exemple, expliquant la notion de pouvoir, H.T. Odum (1971, p. 206) écrivait :

" The true powers of individuals, groups and political bodies lie in the useful potential energies that flow under their control. Power does work, gains and manipulates storages of energy, and directs forces. True power with a real energetic basis is a property that has no substitute. If some philosophy of government or some particular idealism attempts a form of control contrary to the hard facts of energy distribution, that system will fail [...]. The energetic laws are as much first principles of political science as they are first principles of any other process on earth. »

Plus complexe et plus quantitative que la métaphore organiciste, la métaphore systémiste s'imposera donc aussi en écologie humaine grâce, cette fois, à des écologues faisant incursion dans le champ des sciences sociales. Tel était le cas de Dansereau.

\section{L'écosystème chez Pierre Dansereau}

Dansereau affirmera souvent que la perspective systémique est la plus pertinente pour décrire et comprendre l'interaction entre les différents règnes de la nature, dont celui de l'espèce humaine. Cette position semble toutefois lui être venue tardivement, alors qu'il développait son écologie humaine. Dans Biogeography, Dansereau utilisait également des concepts du ressort d'autres traditions, dont ceux de « climax » et de "succession », qui avaient par ailleurs évolué depuis leur première énonciation dans les travaux de Clements. Comme pour répondre aux désirs des écologues de trouver des lois générales, et aussi pour contribuer aux théories de la niche qui se développaient en parallèle de l'approche systémique, Dansereau (1957, p. 257) y formulait la « loi de l'inoptimum », qui explique qu' " aucune espèce ne trouve, dans un habitat donné, les conditions optimales pour toutes ses fonctions ». Il effectuait, dans ce même Biogeography, une synthèse des diverses échelles d'appréhension des objets de l'écologie, par exemple en s'attardant sur la bioclimatologie (étude des phénomènes de changement climatique et biotique dans un milieu donné), sur la synécologie (l'analyse des unités multispécifiques et des "sociétés » d'espèce) et sur l'autécologie (étude du métabolisme et du comportement individuel des espèces dans un milieu donné). Ainsi, avant de devenir humaine, l'écologie de Dansereau fut fondamentale. Toutefois, déjà dans cet ouvrage d'écologie qui eut certaines répercussions dans l'écologie nord-américaine de l'époque (Dupont, 2010 ; Hébert, 2006), Dansereau entrevoyait la nécessité de considérer les écosystèmes dans leurs interactions avec l'activité humaine. Ainsi, avec les années 1960 s'ouvrent dans les travaux de Dansereau de nouvelles voies de recherche portant sur "l'escalade de l'impact humain », sur « les dimensions écologiques de l'espace urbain » et sur la notion - d'ailleurs elle aussi métaphorique - de « paysage intérieur ». Dans cette section, nous retracerons ce chemin qui mène de la notion d'écosystème vers la discipline de l'écologie humaine dans la pensée de Dansereau. C'est là que l'écologie systémique aura une influence plus prononcée dans ses travaux.

L'horizon théorique des recherches de Dansereau "repose sur les sciences de l'environnement, ellesmêmes unifiées par une pensée écologique » nécessairement systémique (Dansereau, 1991, p. 10). Son écologie s'oriente alors vers la description des flux de matière, d'énergie et d'information et vers l'analyse de la complexité des systèmes écologiques qui en résultent. Ainsi l'écologue considérait-il plusieurs niveaux de complexité à travers son concept d'écosystème, lui-même illustré par la fameuse «boule de flèches » (Fig. 1). Selon Dansereau (1987, p. 340), l'écosystème est « un espace limité où le cyclage des ressources à travers un ou plusieurs niveaux trophiques est effectué par des agents plus ou moins fixés et nombreux, utilisant simultanément et successivement des processus mutuellement compatibles qui engendrent des produits utilisables à courte ou longue échéance ».

L'écosystème est donc un espace où circulent la matière, l'énergie et l'information. La notion de niveau trophique sert ici de matrice pour illustrer ces processus de circulation. Dansereau identifie six niveaux trophiques, qu'il définit à la fois comme des étapes du cyclage et comme les strates fonctionnelles de l'écosystème. Les niveaux trophiques apparaissent donc à la fois comme des unités temporelles, si l'on considère l'évolution d'un écosystème (qui peut être caractérisé par l'un ou l'autre des niveaux trophiques à un moment donné de son 


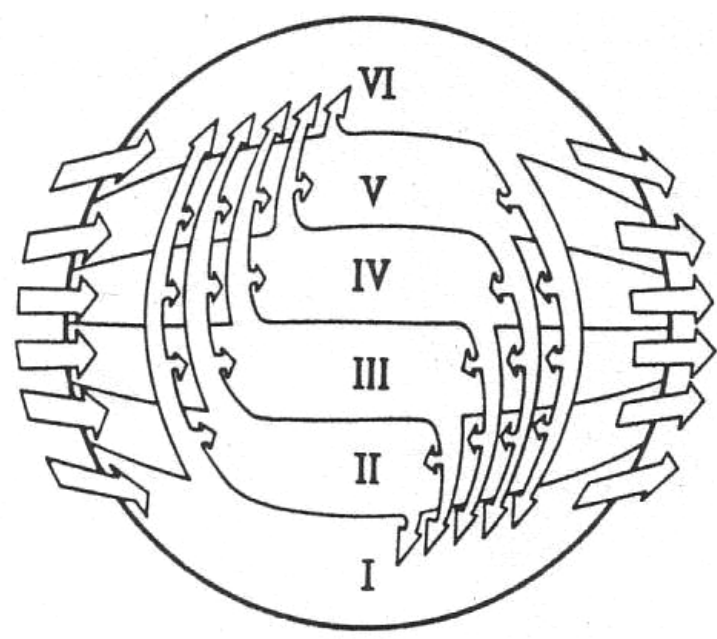

Fig. 1. La «boule de flèches » (source : Dansereau, 1987).

évolution - nous y reviendrons plus loin), et comme des unités spatiales et fonctionnelles qui s'interpénètrent, telles que le sol, les strates herbacées et arbustives où vivent les animaux, etc. Chaque niveau trophique est ainsi caractérisé par un ou plusieurs processus de cyclage de la matière et de l'énergie en présence. Le niveau de la minérotrophie (I) se caractérise par les processus de désagrégation, d'érosion, etc. ; le niveau de la phytotrophie (II), par les processus de photosynthèse, d'enracinement, etc. ; le niveau de la zootrophie herbivore (III), par les processus de phytophagie, de migration, de reproduction, etc. ; le niveau de la zootrophie carnivore (IV), par les processus de prédation, de migration, etc. ; le niveau de l'investissement, ou technotrophie (V), par les processus de mise en réserve, de labour, de construction, d'urbanisation, etc. ; le niveau du contrôle, ou noötrophie (VI), par les processus d'aménagement, de planification et de financement.

Le schéma de la « boule de flèches » permet d'illustrer les principaux niveaux trophiques caractérisant un milieu (avec les strates dans la figure 1), les principaux agents qui participent au cyclage des ressources et selon quels processus (avec les flèches verticales), ainsi que les échanges qui s'établissent avec d'autres écosystèmes (avec les flèches latérales) ${ }^{6}$. Il permet de découper et de décrire des unités d'analyse dont les frontières ont l'avantage d'être malléables et l'échelle variable, allant du parc urbain à la forêt boréale. Ainsi, les écosystèmes se distinguent les uns des autres, d'une part, par leur relative autonomie ou dépendance et, $d^{\prime}$ autre part, par les différentes répartitions des charges trophiques qu'on y trouve. Un désert,

6 En utilisant des couleurs ou divers tons de gris, Dansereau (1987) remplit les différents espaces (niveaux trophiques) de la « boule de flèches » selon l'écosystème qu'il veut décrire : pour l'érablière, le niveau II sera plus foncé que les autres; pour la mégapole, les niveaux I, V et VI seront les plus foncés, etc. par exemple, est dominé par la minérotrophie ; une érablière, par la phytotrophie; une ville industrielle, par l'investissement; une mégapole, par le contrôle, etc. Dansereau (1987) insiste souvent sur le fait qu'un écosystème urbain, comme tout autre écosystème, peut très bien être illustré grâce à une représentation adéquate de la «boule de flèches ». Les écosystèmes humains sont ainsi caractérisés par la prédominance des niveaux trophiques de l'investissement et $\mathrm{du}$ contrôle, deux niveaux trophiques sur lesquels il importe d'insister pour comprendre le pendant humain de l'écologie de Dansereau.

L'investissement (niveau V : technotrophie) correspond à «la mise en réserve de matériaux ou à la construction d'artefacts qui ont un usage à long terme pour le maintien d'une certaine condition ou pour le cyclage d'une ou de plusieurs ressources » (Dansereau, 1973, p. 73). Il s'agit en réalité d'une réorganisation des ressources provenant des autres niveaux trophiques en vue d'une utilisation ultérieure ou continue. Si plusieurs plantes et animaux en sont capables, ce qui caractérise l'investissement typiquement humain est son intensité, comme en témoignent les villes, les routes et les infrastructures en général, que l'on peut interpréter comme des assemblages de matières minérales et synthétiques jouant un rôle structurant dans le stockage et la circulation de l'énergie, des produits et aussi des agents, les humains. Quant au niveau trophique du contrôle (niveau VI : noötrophie), il est incarné par les systèmes commerciaux, administratifs, économiques, politiques, religieux et culturels, qui « déterminent non seulement la qualité, la quantité et la périodicité d'importation de pierres et de fer, de légumes et de viande [...] », mais qui «font [aussi] sentir leur impact bien au-delà de la ville » (ibid., p. 90). Le contrôle est un niveau informationnel et communicationnel qui non seulement ordonne la circulation de matière et d'énergie au sein d'un écosystème, mais qui, s'il est très puissant, peut également ordonner la circulation de matière et d'énergie entre des écosystèmes lointains. Ainsi, par exemple, des décisions administratives ou économiques prises tout en haut d'une tour à Montréal pourront avoir des conséquences sur des écosystèmes marins de l'Arctique. À cause de leur forte densité d'investissement et de contrôle, les écosystèmes humains sont sérieusement dépendants de tous les autres écosystèmes ; mais, en retour, ils exercent un pouvoir considérable sur ceux-ci en contrôlant à distance les processus d'exploitation et de cyclage des ressources.

Marquons ici un temps d'arrêt afin de considérer la place de l'humain dans l'écologie de Dansereau. L'idée d'un « agent » dont la principale activité est le « cyclage des ressources » au sein de "niveaux trophiques » évoquerait certes une vision froide et désincarnée de l'être humain. C'est d'ailleurs peut-être le propre des métaphores naturalistes que de se placer à un niveau d'analyse si «macroscopique " que l'on perd de vue l'humanité 
des individus et des collectivités. Pourtant, en humaniste résolu, Dansereau s'ouvrira à cette contradiction et tentera $\mathrm{d}^{\prime} \mathrm{y}$ répondre à travers son écologie humaine. Il formulera dès lors des propositions sur l'indispensable compréhension des dimensions symboliques de la vie sociale pour l'analyse des écosystèmes, puisque, par exemple, «des blocages [psychologiques ou culturels] ont des effets sur le cyclage des ressources tout à fait semblables par leur force inhibitrice à l'inondation, à la disette, à l'excès de combustible ou à la congestion du transport » (Dansereau, 1973, p. 81). À partir d'ici, l'investissement et le contrôle nous mènent donc sur le terrain d'une écologie humaine plus consciente de la dimension symbolique de la vie sociale.

\section{L'écologie humaine et le paysage intérieur}

L'écologie humaine prétend généralement décrire en termes écologiques des problématiques traditionnellement réservées aux sciences humaines et sociales. Dansereau (1987, p. 334) admettra volontiers que « l'écologie humaine est fondée sur une hypothèse de travail qui emprunte ses concepts et une partie de sa méthodologie à l'écologie animale et même végétale ». Cet emprunt se justifie par le fait que l'écologie humaine doit décrire dans un langage commun les diverses modalités des interactions entre société et écosystème. C'est en tout cas la direction qu'emprunte Dansereau en proposant d'autres schémas qui illustrent « l'escalade de l'impact humain » et « la satisfaction des besoins » et dans lesquels il formule explicitement le projet de saisir des processus proprement sociaux en les intégrant au modèle écosystémique. Ce sont ces exercices qui semblent le mieux révéler les limites de la métaphore systémiste face aux défis que représente l'analyse de la dimension symbolique de la vie sociale et du rapport à la nature.

Dansereau (1970, p.683) affirme qu'envisager l'humain en tant qu'élément de la nature « devrait permettre de déterminer la qualité et la force de son action », et c'est cette hypothèse qui guide sa représentation en six phases de l'escalade de l'impact humain. En réalité, il s'agit ici de décrire l'histoire du niveau trophique du contrôle chez l'humain, c'est-à-dire du " pouvoir sur l'habitat dans son ensemble » (Dansereau, 1973, p. 99). L'histoire de ces six phases se fonde notamment sur la dimension temporelle de la notion de niveau trophique abordée plus haut. Nous les résumons ici en quelques lignes ${ }^{8}$ :

\footnotetext{
7 Pour reprendre le terme utilisé par Joël de Rosnay (1977), lui-même important adepte de telles approches.

8 Ces six phases de l'impact humain ont été développées de manière progressive par Dansereau à partir de Biogeography (1957), puis dans de nombreux textes, notamment Dansereau $(1970,1973,1980$ et 1987).
}

1. La phase indigène est complètement dominée par la sélection naturelle et, si l'humain y est présent, il ne contrôle pas les processus écologiques de base.

2. Avec la phase colligène, celui-ci commence à mieux tirer profit des ressources de l'environnement grâce à la cueillette et à la capture, mais il n'a encore qu'un impact marginal.

3. Ensuite, vient la phase agrigène, où l'humain n'occupe pas encore très densément le territoire, mais commence à transformer les milieux grâce à la culture de plantes, à l'élevage et au pâturage. Un contrôle s'exerce alors sur les niveaux II à IV (phytotrophie et zootrophie) et, plus modérément, sur le niveau $\mathrm{V}$ (investissement).

4. La phase fabrigène reflète un contrôle beaucoup plus déterminant sur tous les niveaux trophiques. On y trouve des installations ponctuelles (usines) ou rassemblées (parcs industriels, villes) qui s'appliquent à la transformation massive des ressources et qui «sont le plus souvent efficacement tamponnées contre les processus naturels » (Dansereau, 1987, p. 351).

5. Ensuite, le commerce à grande échelle (et donc l'exportation de contrôles), l'architecture, la construction dense et la concentration des décisions font des milieux humains de la phase urbigène de véritables puissances de contrôle des autres écosystèmes.

6. Enfin, la phase cybernigène voit les activités humaines se concentrer de plus en plus sur la création, la diffusion d'information et de commandes, la pensée et l'intellect. Cette phase est «essentiellement psychosociale en ce qu'elle engage d'une façon nouvelle la perception du savoir qu'ont les individus et en ce qu'elle leur offre en même temps des moyens sans précédents d'élargir leur expérience » (Dansereau, 1973, p. 112).

Les mots "perception ", "savoir » et " expérience », ici, sont importants parce qu'ils renvoient à la dimension symbolique de la vie sociale. Pour Dansereau, la phase cybernigène présente une occasion de s'acheminer vers une planification plus rationnelle et plus universelle de l'aménagement. Une telle planification, peut-on penser, ne saurait s'accomplir sans un travail sur les représentations, les valeurs et, plus largement, sur toute cette dimension symbolique de la vie sociale, ce qui demande alors de faire appel à la sociologie et aux autres sciences sociales. Mais ce premier appel aux sciences sociales est mitigé : il repose sur l'idée que la rationalité requise pour la planification écologique est un principe universel, une donnée découlant tout naturellement des besoins humains. C'est dans cette optique que Dansereau élabore son « gâteau de l'environnement » (Fig. 2) : il s'agit d'un schéma représentant les besoins des individus (subdivisés en six classes: physiologiques, psychologiques, sociaux, économiques, politiques et religieux), de la société et de l'espèce.

Dansereau pose quatre degrés de satisfaction (les quatre cercles concentriques) pour tous ces besoins : la 


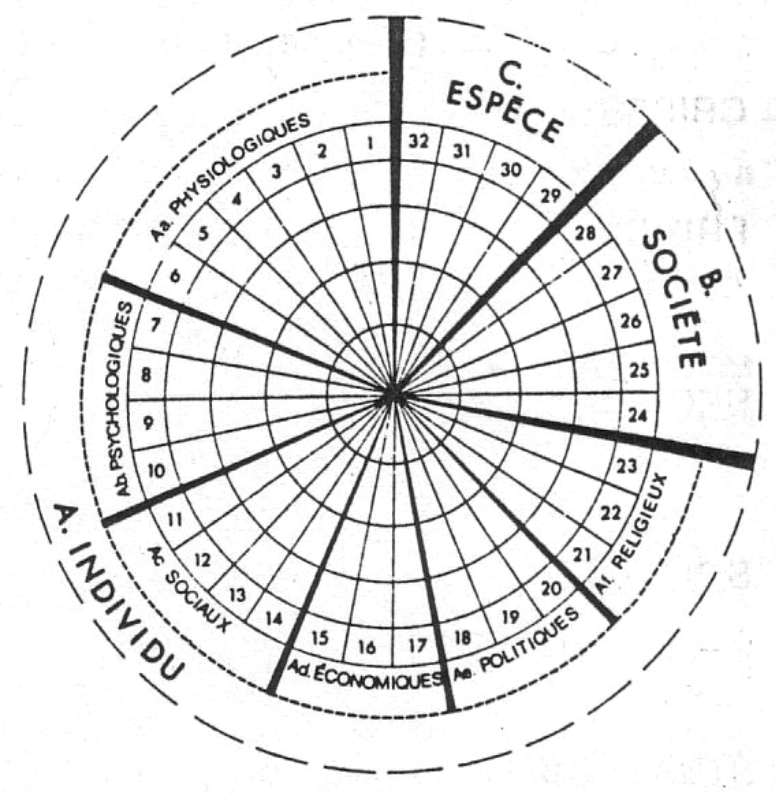

Fig. 2. Le «gâteau de l'environnement » (source : Dansereau, 1987).

privation, la nécessité, le comblement et le surplus. En ajoutant des tons de gris dans les cases du schéma, il est possible d'y représenter quels niveaux trophiques sont aptes à procurer la satisfaction des besoins, ou encore les forces qui entravent cette satisfaction. Ce schéma, si on l'applique par exemple à deux écosystèmes urbains, peut très bien servir à détecter les inégalités ou encore à constater l'impact de la satisfaction des besoins sur les ressources. En fin de compte, le "gâteau de l'environnement » constitue une « toile de fond [sur laquelle] on pourra projeter des conflits et des satisfactions » (Dansereau, 1990, p. 20). Dans la perspective de l'intervention et de la planification écologique, nous avons là un autre outil pouvant s'avérer utile.

Mais cette tentative de Dansereau pour aborder la question des représentations sociales reste essentiellement descriptive et demeure muette à l'égard des opportunités de développement culturel que suppose la phase cybernigène, et surtout de la nécessaire compréhension des aspects plus symboliques du changement social. Comment, en demeurant dans une perspective aussi naturaliste, peut-on envisager la "construction sociale de la nature »? Dansereau est conscient de cette limite. Il poursuivra alors une piste à la fois plus ouverte et imprécise, à la fois poétique et engagée, pour aborder la dimension symbolique du rapport entre les sociétés humaines et leurs écosystèmes. Pour comprendre ultimement les problèmes écologiques, dira-t-il, il nous faut comprendre notre « paysage intérieur».
Nous avons déjà souligné que, pour Dansereau, l'esprit humain est traversé de tensions et d'émotions qui déterminent considérablement à la fois l'activité et les besoins, et donc la manière de façonner l'environnement. L'adaptation à l'environnement, admet Dansereau, se fait souvent grâce à des réponses symboliques plutôt que techniques ou matérielles. Ces données, il est impossible de les illustrer sur les schémas de la «boule de flèches » et du « gâteau de l'environnement ». D'où l'importance d'analyser les perceptions, les valeurs et les croyances sur l'environnement, c'est-à-dire les « représentations sociales » de l'environnement - le paysage intérieur. Il faut, dit-il, « nous poser des questions au sujet de la qualité et de la sélectivité de ces perceptions d'une part, et de la force toujours croissante de son pouvoir d'intervention d'autre part » (Dansereau, 1990, p. 15). Mais l'écologie humaine est-elle capable de répondre au défi ? Peut-on vraiment décrire et comprendre la dimension symbolique de la relation humaine à l'environnement en termes écologiques ? L'expérience de la première écologiehumaine du groupe de Chicago commande la prudence. La deuxième écologie humaine, dont Pierre Dansereau fut un représentant, aboutit à un appel à l'intervention de la sociologie, de l'anthropologie et de la psychologie, comme pour admettre que cette tâche n'est peut-être pas celle de l'écologie humaine.

\section{Conclusion : éthique et paysage intérieur}

L'écologie humaine de Dansereau entreprend d'intégrer les processus sociaux dans un cadre explicatif systémiste et naturaliste. Or, cette démarche se butte éventuellement à la difficulté de traduire la complexité des représentations sociales et de la « construction sociale de la nature » dans un langage qui leur est étranger. $C^{\prime}$ est pour pallier ce problème que Dansereau propose la belle métaphore du paysage intérieur, notion qui rassemble du même coup la perspective éthique d'un changement social conscient et planifié.

Les modèles développés par Dansereau aboutissent tous à l'idée que « le repérage de la motivation est quelque chose de passablement important [...] [et que] la perception est donc décidément du ressort de l'écologie, car c'est à son niveau que se trouvent certains blocages qui arrêtent les processus de cyclage et de production » (Dansereau, 1990, pp. 19-20). C'est d'ailleurs pourquoi « la relation de l'imagerie intérieure à la terre des hommes est plus que jamais sujette à une pondération éthique » et que, si l'écologie reste incapable de fournir des valeurs et des principes éthiques, " elle peut nous donner un arrièreplan considérablement amélioré pour un estimé réel et réaliste dela responsabilitéhumaine et sociale »(Dansereau, 1973, p. 137 et 140). Cette préoccupation pour l'éthique, Dansereau l'avait déjà exprimée dans son ouvrage phare 
de 1957. Mais, alors qu'il développe plus complètement son écologie humaine et que s'accumule la sagesse des années, la dimension éthique et normative devient centrale. Au crépuscule de sa carrière, il insistera de plus en plus sur la nécessité d'intégrer au paysage intérieur de l'humanité une philosophie de « joyeuse austérité ».

Peut-être d'ailleurs ce positionnement éthique et normatif est-il un passage obligé pour toutes ces « écologies » qui s'intéressent aux enjeux de la relation entre l'être humain et son environnement. Les métaphores naturalistes, dont nous avons tenté de montrer qu'elles limitent considérablement la capacité de l'écologie à comprendre la dimension symbolique de la vie sociale, ne sont-elles pas elles-mêmes issues d'un positionnement normatif « holiste » qui cherche à trouver les principes unificateurs de la vie sur Terre (Gunnel, 2009) ? L'écologie politique, en Europe, n'est-elle pas encore plus explicitement normative, tout comme l'écologie sociale aux États-Unis ? Il ressort de tout cela que l'écologie, peu importe l'épithète qu'on lui accole, peut difficilement faire l'économie d'une réflexion épistémologique sur les positionnements normatifs et éthiques qu'elle suppose, qu'ils soient métaphoriques ou non. Pierre Dansereau, humaniste centenaire, écologue ayant vu naître sa science, aura aussi eu le génie de poser clairement la question du paysage intérieur à l'écologie humaine.

\section{Remerciements}

L'auteur tient à remercier le Conseil de recherche en sciences humaines du Canada (CRSH), programme de bourse postdoctorale, pour le soutien reçu au cours de la rédaction de cet article. Des conversations avec Jean-Guy Vaillancourt, professeur à l'Université de Montréal, et Raymond Murphy, professeur émérite à l'Université d'Ottawa, ont grandement contribué au développement des idées qui y sont exposées. L'auteur est également reconnaissant envers deux évaluateurs anonymes dont les commentaires ont permis d'améliorer cet article.

\section{Références}

Acot, P., 1994. Histoire de l'écologie, Paris, Presses universitaires de France.

Catton, W.R., Jr., Dunlap, R.E., 1980. A new ecological paradigm for post-exuberant sociology, The American Behavioral Scientist, 24, 1, 15-47.

Clements, F.E., 1928. Plant Succession and Indicators: A Definitive Edition of Plant Succession and Plant Indicators, New York, Hafner Press.

Dansereau, P., 1957. Biogeography: An Ecological Perspective, New York, The Ronald Press Company.
Dansereau, P., 1970. L'écologie et l'escalade de l'impact humain, Revue internationale des sciences sociales, XXII, 4, 683-706.

Dansereau, P., 1973. La Terre des hommes et le paysage intérieur, Montréal, Leméac.

Dansereau, P., 1980. Écosystème, rythme de la vie urbaine et aménagement du temps, Apprentissage et socialisation, 3, 2, 88-106.

Dansereau, P., 1987. Les dimensions écologiques de l'espace urbain, Cahiers de géographie du Québec, 31, 84, 333-395.

Dansereau, P., 1990. Les forces de la nature, les réponses de la culture, Vie des arts, 141, 14-21.

Dansereau, P., 1991. L'Envers et l'endroit : le besoin, le désir et la capacité, Saint-Laurent, Fides/Musée de la civilisation.

Dansereau, P., Brunet, N., Pivot, A., 2004. Pierre Dansereau, le gentilhomme décodeur et iconoclaste de l'écologie, Natures Sciences Sociétés, 12, 1, 75-82.

Deléage, J.-P., 1991. Une histoire de l'écologie, Paris, La découverte.

Drouin, J.-M., 1993. L'Écologie et son histoire : réinventer la nature, Paris, Flammarion.

Dumesnil, T., 1981. Pierre Dansereau : l'écologiste aux pieds nus, Montréal, Nouvelle optique.

Dunlap, R.E., 2008. Promoting a paradigm change. Reflections on early contributions to environmental sociology, Organization \& Environment, 21, 4, 478-487.

Dupont, L., 2010. Autour des 100 ans de Pierre Dansereau. Cinq générations d'écologues, Découvrir, 31, 2, 38-55.

Eder, K., 1996. The Social Construction of Nature: A Sociology of Ecological Enlightenment, London, SAGE Publications.

Grafmeyer, Y., Joseph, I. (Eds), 1979. L'École de Chicago: naissance de l'écologie urbaine, Paris, Éditions du Champ urbain.

Gunnel, Y., 2009. Écologie et société : repères pour comprendre les questions d'environnement, Paris, Armand Colin.

Hébert, Y., 2006. Une histoire de l'écologie au Québec : les regards sur la nature des origines à nos jours, Québec, Les Éditions GID.

Lafontaine, C., 2004. L'Empire cybernétique : des machines à penser à la pensée machine, Paris, Le Seuil.

Latour, B., 1997. Nous n'avons jamais été modernes: essai d'anthropologie symétrique, Paris, La Découverte.

Lentin, F., 1977. Écologie et biologie, in Archand, P., Chauvenet, A., Lage, É., Lentin, F., Nève, P., Vignaux, G., Discours biologique et ordre social, Paris, Le Seuil, 206-240.

McKensie, R.D., 1979 [art. orig. 1925]. L'approche écologique dans l'étude de la communauté humaine, in Grafmeyer, Y., Joseph, I. (Eds), L'École de Chicago : naissance de l'écologie urbaine, Paris, Éditions du Champ urbain, 149-166.

Odum, H.T., 1971. Environment, Power, and Society, New York, John Wiley \& Sons, Inc.

Rosnay, J. de, 1977. Le Macroscope : vers une vision globale, Paris, Le Seuil.

Tansley, A., 1935. The use and abuse of vegetational concepts and terms, Ecology, 16, 3, 284-307.

UQAM (Université du Québec à Montréal), 2010. Pierre Dansereau, écologiste : rencontre de l'homme avec la nature. Exposition en ligne: http://www.archives-expopd.uqam.ca/ (consulté le 13 juillet 2010).

Vaillancourt, J.-G., 1999. Pierre Dansereau, écologue, écosociologue et écologiste, Sociologie et sociétés, XXXI, 2, 191-193. 